\title{
Archéologie(s) de la traduction, dir. G. HENROT SOSTERO
}

\section{Fabio Scotto}

\section{(2) OpenEdition \\ Journals}

\section{Edizione digitale}

URL: https://journals.openedition.org/studifrancesi/45448

DOI: $10.4000 /$ studifrancesi. 45448

ISSN: 2421-5856

\section{Editore}

Rosenberg \& Sellier

\section{Edizione cartacea}

Data di pubblicazione: 1 août 2021

Paginazione: 400-401

ISSN: 0039-2944

\section{Notizia bibliografica digitale}

Fabio Scotto, «Archéologie(s) de la traduction, dir. G. henrot sostero», Studi Francesi [Online], 194 (LXV I

II) | 2021, online dal 01 septembre 2021, consultato il 15 octobre 2022. URL: http://

journals.openedition.org/studifrancesi/45448 ; DOI: https://doi.org/10.4000/studifrancesi.45448

Questo documento è stato generato automaticamente il 15 octobre 2022.

\section{(c) (i) ()}

Creative Commons - Attribuzione - Non commerciale - Non opere derivate 4.0 Internazionale - CC BYNC-ND 4.0

https://creativecommons.org/licenses/by-nc-nd/4.0/ 


\title{
Archéologie(s) de la traduction, dir. G. HENROT SOSTERO
}

\author{
Fabio Scotto
}

\section{NOTIZIA}

Archéologie(s) de la traduction, dir. G. HENROT SOSTERO, Paris, Classiques Garnier, 2020, «Translatio» 3, $303 \mathrm{pp}$.

1 È qui raccolta, in una ricca e varia miscellanea, una serie di studi che indagano, da una prospettiva che fonde la traduttologia alla genetica testuale, alla linguistica e alla psicolinguistica, l'elaborazione del processo traduttivo dalla duplice prospettiva della genesi dell'originale e della genesi della sua successiva traduzione. Nel suo «Avantpropos» (pp. 7-13), la curatrice Geneviève HENROT SOSTERO delinea lo stato dell'arte delle ricerche di ordine genetico attorno alla traduzione oggi, con un ampio ventaglio di utili rimandi bibliografici e documentari, anche presentando le linee di ricerca dei contributi contenuti nel volume. Ne è ampia espansione, il testo liminare a sua firma della «Première partie. Traduction et brouillons d'auteurs» dal titolo Fondéments théoriques et méthodologiques pour une génétique de la traduction. Concepts, méthodes, visées (pp. 17-56), nel quale precisa anche il senso del titolo del volume nella cui "s", posta tra parentesi, del plurale «archéologie(s)», si esprime il desiderio di proporre «une approche disciplinaire (la génétique de la traduction) inspirée d'une pluralité d'autres approches de l'objet "écrit": les sciences cognitives, les sciences du langage, la traductologie, la philologie, la stylistique, la génétique de la création (littéraire et autres)...». Dopo aver evidenziato le differenze tra l'approccio filologico e genetico, riconoscendo al secondo un maggiore dinamismo, s'addentra nella filogenesi e nell'ontogenesi degli studi dei manoscritti, per poi soffermarsi sugli esiti della critica genetica nella determinazione delle fasi di progressiva strutturazione del testo, anche grazie all'apporto della tecnologia, fino a definirne gli obiettivi attraverso una categorizzazione delle tipologie avantestuali da una prospettiva che tende a valorizzare 
gli apporti dello strutturalismo linguistico applicato alla genetica e una pragmatica traduttologica tesa a dialogare proficuamente con la linguistica genetica per l'analisi di testi non solo letterari.

Completano questa sezione del volume i saggi di Florence PELLEGRINI (Variations sur un jardin. Logique narrative et orthonymie dans cinq traductions italiennes de l'épisode horticole de "Bouvard et Pécuchet", pp. 57-71), che studia le catene causali di alcune versioni italiane basate su uno stadio genetico del testo originale; di David ELDER (“L'Ange" de Valéry. Esquisse d'une étude génétique et traductologique, pp. 73-95), che ricostruisce, alla luce del pensiero traduttivo di Valéry, la genesi del testo per poi metterlo in relazione con la sua traduzione al fine di mostrare come a suo modo ogni studio genetico sia di per se stesso una forma di traduzione. Sempre a Valéry è dedicato lo studio di Jacqueline COURIER-BRIÈRE (Traduire ou «mettre nos pas sur les vestiges de ceux de l'auteur». Valéry en arabe, pp. 97-114), la quale esplora il dossier del traduttore della Jeune Parque Édouard Rouaud Tarabay al fine di soppesarne il lavoro nelle sue valenze metalinguistiche per evidenziarne le conseguenze minute nelle opzioni adottate, dalla punteggiatura alla componente intertestuale e costrittiva dovuta alla sintesi intrinseca dell'idioma traducente.

3 La seconda parte della miscellanea "Génèse d'une pensée traductologique» propone un saggio di Solange ARBER che incrociando traduttologia e genetica testuale rivela l'apporto fornito alla critica della traduzione dallo studio delle minute del traduttore (L'écriture de la traduction. Les brouillons d'Elmar Topoven pour la traduction de "Djinn", pp. 117-128), mentre Viviana AGOSTINI-OUAFI (Génèse et exégèse par André Pézard de sa traduction de Dante, pp. 129-142) si sofferma sul modo in cui Pézard illustra filologicamente il proprio processo genetico di traduzione dell'opera dantesca (con ricorso ai TAP'S, acronimo di Think-Aloud Protocols), Il contributo di Chiara ELEFANTE (Traduire des essais sur la poésie d'Yves Bonnefoy, pp. 143-156) propone invece un interessante esercizio di autoanalisi delle proprie versioni di alcuni saggi di Bonnefoy, occasione per ricostruire la fortuna editoriale italiana dell'Autore, nel Meridiano Mondadori (L'opera poetica, 2010) nel quale esse furono inserite, con particolare riferimento alla riformulazione lessicale, alla costruzione della frase, alla forma interrogativa e alla punteggiatura, tutti aspetti peculiari dello stile del poeta di Tours.

4 La terza parte «Anamnèses» si apre con un contributo di Simona Pollicino (Énumération elliptique et syntaxe nominale dans les "Motets" d'Eugenio Montale traduits par Philippe Jaccottet, pp. 159-173), incentrato sulla resa formale di taluni procedimenti retorici secondo la nota modalità improntata al sermo humilis del poeta di Grignan da poco scomparso, cui segue l'articolo di Vanda MIKŠıć (Traduire Georges Perec en français?, pp. 175-193) riguardo alla singolare vicenda di un originale perechiano perduto ricostruito da una traduzione bosniaca, donde una "retrotraduzione" frutto di un passo retrospettivo nell'archeologia del testo. Con Beate LANGENBRUCH (Pérégrinations transeuropéennes et transatlantiques de la matière épique médiévale de "Fierabras". Enjeux de traduction, entre la France et le Brésil, pp. 195-213) s'affronta il problema dell'adattamento traduttivo di un testo epico medievale nella prospettiva di una retro-traduzione francese che tenga conto di tutte le variabili storico-culturali di contesto in ambito extraeuropeo.

5 Nella quarta parte "Observatoires de l'activité traduisante», apprezzabile appare la sensibilità mostrata da Marie-Claire DURAND-GUIzIOU ( $\mathrm{La}$ traduction poétique, questionnement et plaisir esthétique, une gageure. La traduction des "Roses d'Hercule" du poète 
espagnol Tomás Morales, pp. 217-232) nel conciliare senso e suono nella sua resa del portato lirico dell'opera del poeta ispanico prescelto; dal canto suo, Marina GIAVERI ( $L a$ traduction face à la critique génétique, pp. 233-244) dà conto, con dovizia e pertinenza, delle linee direttrici della critica genetica in Italia e in Francia, con particolare riferimento alla traduzione, che apre alla genetica testuale sviluppi di ampia potenzialità scientifica. Infine Madeleine STRATFORD et Mélanie RIVET (Dans la tête de la traductrice. L'influence des outils sur la créativité en traduction littéraire, pp. 245-256) rivelano l'utilità della consultazione delle fonti anche attraverso lo studio dell'osservazione del traduttore all'opera, al fine di soppesare la conseguente creatività delle soluzioni da questi adottate.

6 In Appendice una vasta «Bibliographie» (pp. 257-282), un «Index des noms» (pp. 283-288), un «Index des œuvres» (pp. 289-290) e un «Index des concepts» (pp. 291-293). 